\title{
A Simple Synthesis of 2'-Deoxy-3'-Thioinosine and Its Phosphorothioamidite
}

\author{
Qianfu Luo,* Renjun Cheng, Qiu Wang, Shuming Bao \\ Key Lab for Advanced Materials and Institute of Fine Chemicals, East China University of Science and Technology, Shanghai 200237, \\ P. R. of China \\ Fax+86(021)64250597; E-mail: luoqf@ecust.edu.cn
}

Received: 10.07.2012; Accepted after revision: 09.11.2012

\begin{abstract}
O-\{[\operatorname{Bis}(4-$ methoxyphenyl)(phenyl)methyl])-2'-deoxy-3'-thioinosine and its $S$-phosphorothioamidite were prepared in five and six steps, respectively, from commercially available 2 '-deoxyinosine. The key $3^{\prime}$-thio intermediate was synthesized by two sequential configuration inversions: a one-pot oxidation/reduction reaction and an $\mathrm{S}_{\mathrm{N}} 2$ reaction. This intermediate was readily converted into the target compound with a high yield. Compared with other methods, this synthetic strategy has the advantages of brief reaction steps, a relatively high overall yield, and regioselectivity for the configuration inversions. The products might be useful as intermediates for the preparation of new functionalized nucleosides.
\end{abstract}

Key words: stereoselective synthesis, heterocycles, medicinal chemistry, nucleosides

The preparation and functionalization of oligonucleotide analogues have attracted an increasing degree of interest because of the variety of potential applications of such compounds. Oligonucleotide analogues have been widely used, for example, in exploring the catalytic mechanisms of enzymes and ribozymes, ${ }^{1}$ in studying physical and biological properties of nucleic acids and RNA, ${ }^{2}$ in controlling gene expression, ${ }^{3}$ in identifying catalytic metal ions, ${ }^{4}$ and in probing hydrogen-bonding interactions. ${ }^{5}$ The biological, biochemical, physical, and medicinal importance of oligonucleotide analogues has fostered the development of various nucleoside modifications.In general, the two primary strategies that are used to prepare structural modifications of nucleotide analogues involve modification of the nucleobase or modification of the sugar residue, respectively. A classical method for modification of the sugar residues involves the removal or replacement of the oxygen atom in the 3 '-position with a larger, moreelectropositive, sulfur atom. ${ }^{6}$ By this approach, a variety of 3'-S-modified nucleosides containing diverse functionalized bases have been prepared as building blocks and intermediates for oligonucleotides. These compounds have been widely used to mimic RNA, to investigate the mechanistic properties of ribozymes, and to increase the resistance of ribozymes to degradation by nucleases. ${ }^{7}$ However, 3'-thio nucleoside analogues that contain inosine-derived bases have received little attention. ${ }^{8}$

Recently, Piccirilli and co-workers ${ }^{9}$ reported the synthesis and biochemical applications of 2'-O-methyl-3'-thioguanosine. The product was incorporated into oligo-

SYNTHESIS 2013, 45, 0106-0110

Advanced online publication: 11.12.2012

DOI: 10.1055/s-0032-1317713; Art ID: SS-2012-H0579-OP

(C) Georg Thieme Verlag Stuttgart · New York nucleotides to study the mechanism of a ribozyme reaction in Tetrahymena. Inspired by this design, we developed an efficient method for the synthesis of a new inosine-containing 2'-deoxy-3'-thionucleoside analogue $\mathbf{6}$, together with its phosphorothioamidite 7, as shown in Figure 1. This product can act as a general base that can pair with any of the four nucleobases ${ }^{10}$ and it can also match any the four base pairs associated with the hybridization effect in any position. ${ }^{11}$ Furthermore, the design of the synthesis of $\mathbf{6}$ provides an alternative method for the synthesis of nucleoside analogues. We hope that these products will be useful as intermediates and building blocks for the preparation of other functionalized nucleosides.

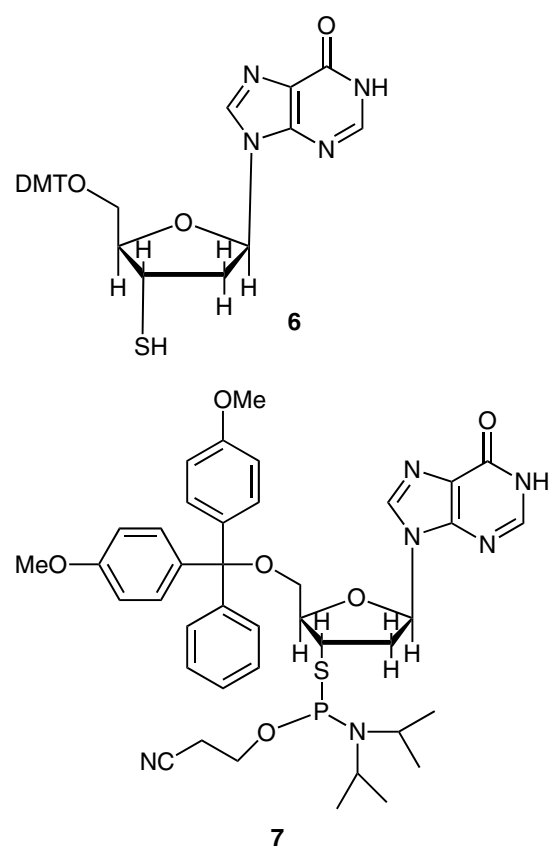

Figure $155^{\prime}-O-\{[\operatorname{Bis}(4-m e t h o x y p h e n y l)($ phenyl)methyl])-2'-deoxy3 '-thioinosine 6 and its $S$-phosphorothioamidite 7

In our synthetic route to the required compounds, the preparation of intermediate 3 (Scheme 1) through inversion of the configuration of the hydroxy group at the 3'position of the sugar moiety is vital. According to the literature, there are several methods for realizing a configurational inversion of a 3'-hydroxy group. First, we examined the approach reported by Challa and Bruice ${ }^{12}$ for the synthesis of $N^{2}$-isobutyryl-2'-deoxy-xylo-guanosine. Treatment 2 '-deoxyinosine (1) with benzoyl chloride in anhydrous pyridine at room temperature gave 5'-Obenzoyl-2'-deoxyinosine (Scheme 1, path a). Treatment of 
this product with 1.5 equivalents of triflic anhydride in dichloromethane containing $10 \%$ pyridine gave 2 -deoxy$x y l o$-inosine, which has an inverted configuration at the 3 '-position. Although we obtained a small amount of this required intermediate, we did not take this route any further because of the low yields and the laborious procedures involved in chromatographic purifications.

We then tried another synthetic route developed by Piccirilli and coworkers ${ }^{9}$ (Scheme 1, path b). In the presence of 4-(N,N-dimethylamino)pyridine, the reaction of 5'-O-[tert-butyl(diphenyl)silyl]-2'-deoxyinosine with triflic chloride in dichloromethane at $0{ }^{\circ} \mathrm{C}$ gave the 3 '-triflate intermediate. Subsequent $\mathrm{S}_{\mathrm{N}} 2$ substitution with sodium bromide in acetone resulted in a product with an inverted configuration at the 3 '-position. However, the conversion was very low, even after prolonged reaction times. This might have been due to the low reactivity and low solubility of 5'-O-[tert-butyl(diphenyl)silyl]-3'-O-triflyl-2'-deoxy-xylo-inosine in the reaction solvent. Finally we opted to perform the configuration inversion at C3' by means of an oxidation-reduction sequence ${ }^{13}$ (Scheme 1, path c).

The complete preparation began with commercially available 2'-deoxyinosine (1). Although oligonucleotide syntheses involving 5 '-silyl protecting groups are known, we choose the bis(4-methoxyphenyl)(phenyl)methyl (4,4'-dimethoxytrityl; Dmt) protecting group for the 5'-O-hydroxyl group, instead of a tert-butyl(dimethyl)silyl or tertbutyl(diphenyl)silyl group, which would have to have been deprotected by treatment with tetrabutylammonium fluoride during subsequent steps. Experiments showed protection with a Dmt group ensured regioselectivity and prevented partial deprotection during oxidation by DessMartin periodinane [1,1,1-triacetoxy-1,1-dihydro-1,2benziodoxol-3(1H)-one]. After reduction by sodium borohydride propan-2-ol, the desired intermediate product $\mathbf{3}$, with the inverted configuration, was obtained in high yield and with high regioselectivity.

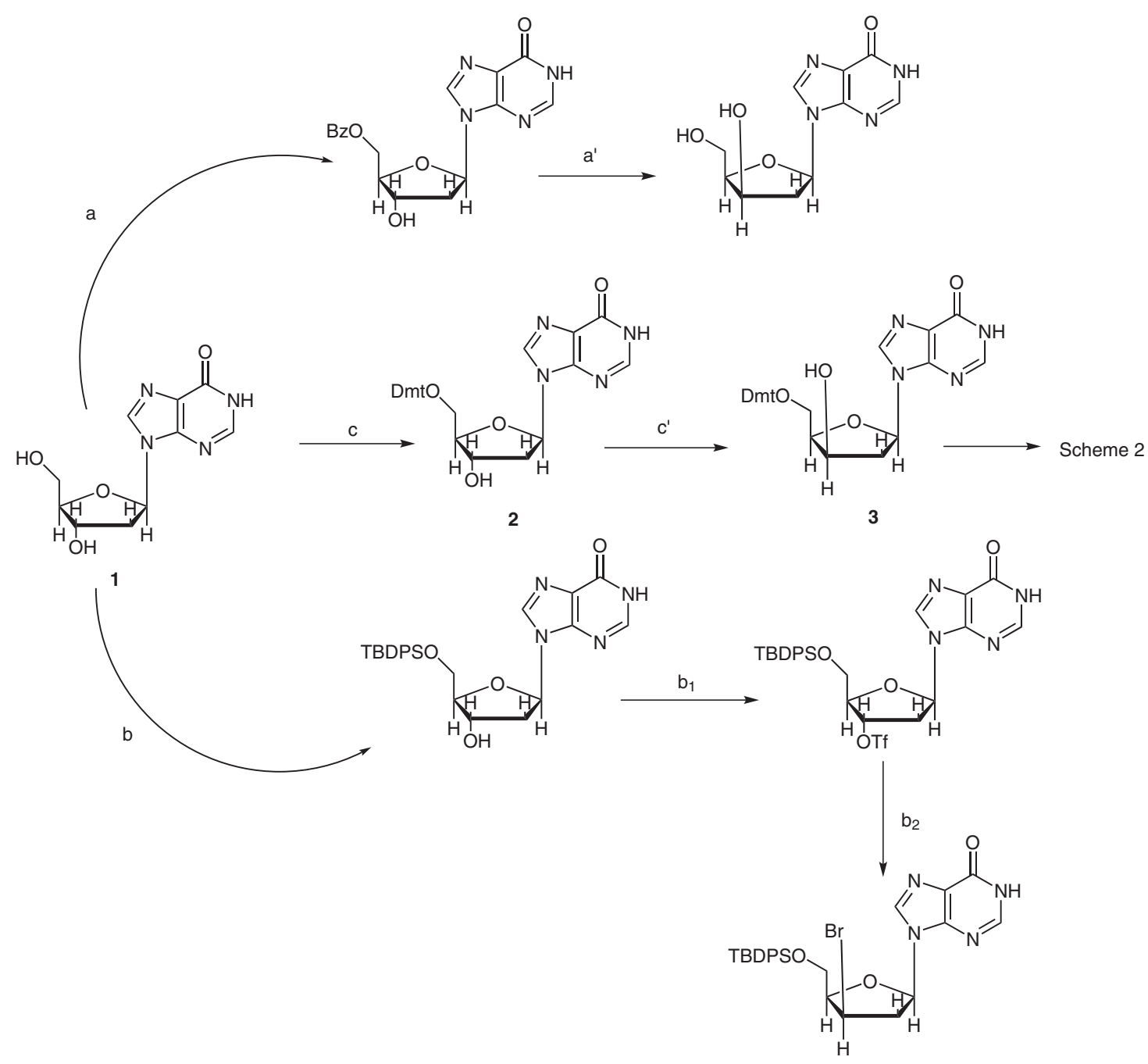

Scheme 1 Reaction conditions: (a) BzCl, py; (a) Tf $2 \mathrm{O}$, py, $\mathrm{CH}_{2} \mathrm{Cl}_{2}$, then $\mathrm{H}_{2} \mathrm{O}$; (b) TBDPSCl, py; $\left(\mathrm{b}_{1}\right.$ ) TfCl, DMAP, $\mathrm{CH}_{2} \mathrm{Cl}_{2}$; $\left(\mathrm{b}_{2}\right) \mathrm{NaBr}$, acetone, reflux; (c) DMTCl, pyridine, ; (c') 1. Dess-Martin periodinane, $\mathrm{CH}_{2} \mathrm{Cl}_{2} ; 2$. $i$ - $\mathrm{PrOH}, \mathrm{NaBH}_{4}$, acetone, $-45^{\circ} \mathrm{C}$. Dmt $=4,4^{\prime}$-dimethoxytrityl. 
Next, we used an $O$-mesyl group as a leaving group and potassium thioacetate as a nucleophile to realize the second inversion at the 3 '-position (from 4 to $\mathbf{5}$ ). In this procedure, compound $\mathbf{3}$ was converted into compound $\mathbf{4}$ by treatment with mesyl chloride in pyridine. Compound $\mathbf{4}$ was treated with potassium thioacetate in $N, N$-dimethylformamide at $60^{\circ} \mathrm{C}$ for 24 hours to give the thioacetate intermediate 5 (Scheme 2).

The target phosphorothioamidite 7 was efficiently obtained in two steps (Scheme 2). First, thioacetate 5 was deprotected by reduction with lithium aluminum hydride to give the sulfanyl derivative $\mathbf{6}$ in high yield $(91.5 \%)$. A solution of $\mathbf{6}$ in dichloromethane was stirred with $N, N$-diisopropylethylamine and 2-cyanoethyl diisopropylamidochloridophosphite at room temperature for three hours to give the target compound 7 in an overall yield of $18.1 \%$.

Compared with literature methods, this synthetic design has advantages of brief reaction steps, a relatively high overall yield, and regioselectivity in the configurationinversion steps. The products should be useful as building blocks and intermediates for the preparation of new functionalized nucleosides.

All chemicals were obtained commercially and used as received unless otherwise mentioned. DMF was dried over $\mathrm{MgSO}_{4}$ and distilled. Pyridine was refluxed over $\mathrm{KOH}$ and then distilled. $\mathrm{CH}_{2} \mathrm{Cl}_{2}$ was heated with $\mathrm{CaH}_{2}$ for $6 \mathrm{~h}$, decanted, and then distilled. Solvents and liquid reagents were introduced from oven-dried microsyringes. TLC analyses were carried out on silica gel 60 F254, and spots were examined under UV radiation. Column chromatography was carried out on silica gel (200-300 mesh).

${ }^{1} \mathrm{H}$ and ${ }^{13} \mathrm{C}$ NMR spectra were recorded on a Bruker AM-500 spectrometer. All mass spectrometric analyses were performed on a ThermoStar mass spectrometer.

\section{5'-O-[Bis(4-methoxyphenyl)(phenyl)methyl]-2'-deoxyinosine $(2)^{14}$}

A stirred soln of 2'-deoxyinosine $(1 ; 0.252 \mathrm{~g}, 1.0 \mathrm{mmol})$ in anhyd pyridine $(10 \mathrm{~mL})$ was treated with DMTCl $(0.406 \mathrm{~g}, 1.2 \mathrm{mmol})$. After $19 \mathrm{~h}, \mathrm{MeOH}(2 \mathrm{~mL})$ was added and stirring was continued for 5 min. The mixture was then transferred into $\mathrm{CH}_{2} \mathrm{Cl}_{2}(20 \mathrm{~mL})$ and the soln was washed successively with $\mathrm{H}_{2} \mathrm{O}(2 \times 20 \mathrm{~mL})$ and brine $(2 \times$ $20 \mathrm{~mL})$ then dried $\left(\mathrm{Na}_{2} \mathrm{SO}_{4}\right)$, filtered, and concentrated under vacuum. The resulting yellow oil was purified by chromatography [silica

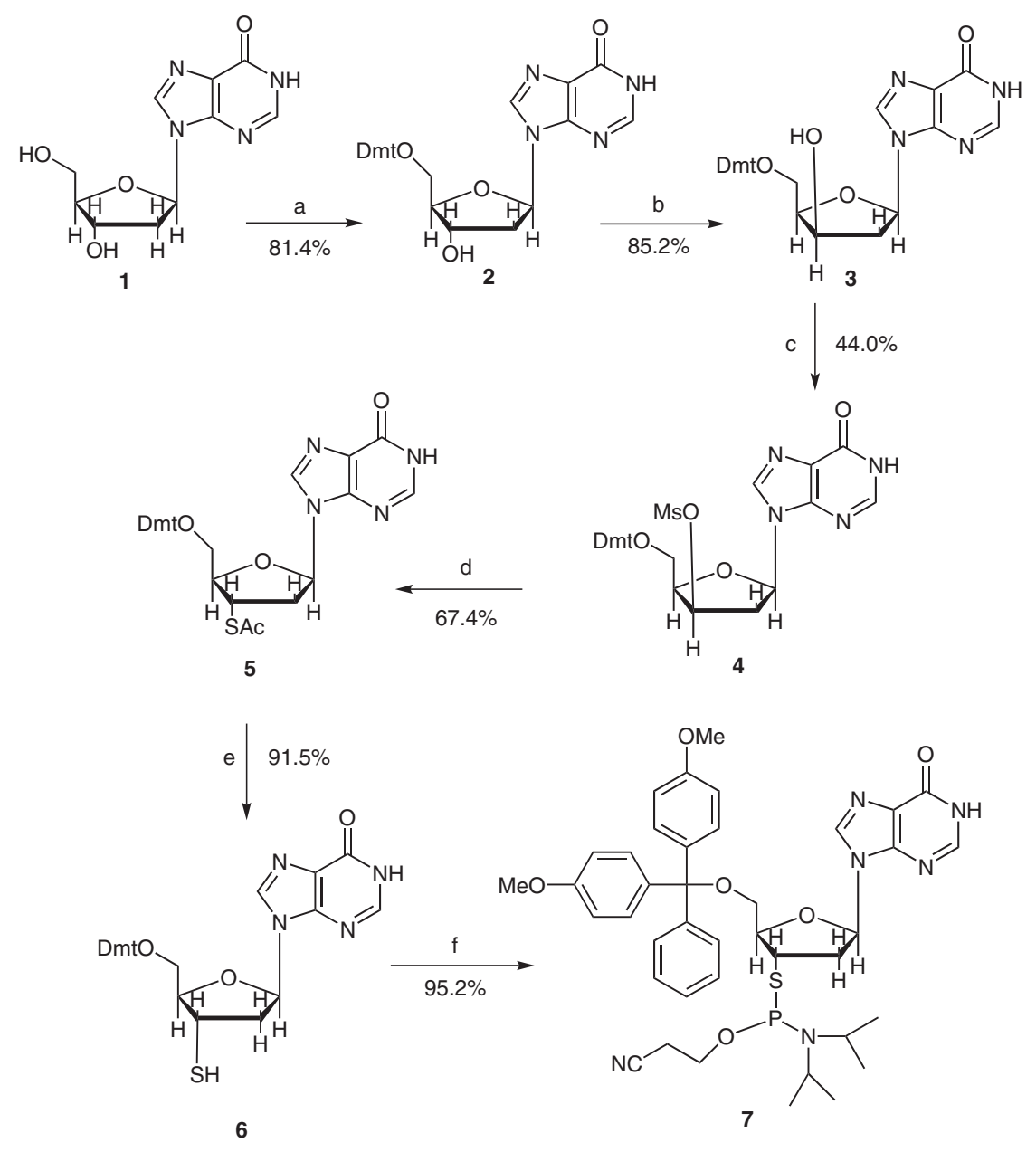

Scheme 2 Reaction conditions: (a) DmtCl, py, r.t., 19 h (81.4\%); (b) (1) Dess-Martin periodinane, $\mathrm{CH}_{2} \mathrm{Cl}_{2}$, r.t. 3 h; (2) $i$ - $\mathrm{PrOH}_{\text {, }} \mathrm{NaBH}$, acetone, $-45^{\circ} \mathrm{C}$, $13 \mathrm{~h}(85.2 \%)$; (c) MsCl, $i$-PrOH, r.t., $12 \mathrm{~h}$ (44.0\%); (d) KSAc, DMF, $60{ }^{\circ} \mathrm{C}$, 24 h $(67.4 \%)$; (e) LiAlH 4 , THF-HOAc, argon, r.t. 3 h (91.5\%); (f) $i-\mathrm{Pr}_{2} \mathrm{NP}(\mathrm{Cl}) \mathrm{O}\left(\mathrm{CH}_{2}\right)_{2} \mathrm{CN}$, DIPEA, $\mathrm{CH}_{2} \mathrm{Cl}_{2}$, r.t., 3 h (95.2\%). 
gel, $\left.\mathrm{CH}_{2} \mathrm{Cl}_{2}-\mathrm{EtOH}(20: 1)\right]$ to give a white solid; yield: 0.451 $(81.4 \%)$

${ }^{1} \mathrm{H}$ NMR (400 MHz, DMSO- $\left.d_{6}\right): \delta=12.37(\mathrm{~s}, 1 \mathrm{H}), 8.19(\mathrm{~s}, 1 \mathrm{H})$, $7.99(\mathrm{~s}, 1 \mathrm{H}), 7.34-6.77(\mathrm{~m}, 13 \mathrm{H}), 6.34\left(\mathrm{dd}, J_{1}=4.0 \mathrm{~Hz}, J_{2}=12 \mathrm{~Hz}\right.$, $1 \mathrm{H}), 5.38(\mathrm{~d}, J=4 \mathrm{~Hz}, 1 \mathrm{H}), 4.42(\mathrm{~m}, 1 \mathrm{H}), 3.97\left(\mathrm{dd}, J_{1}=4 \mathrm{~Hz}, J_{2}=\right.$ $12 \mathrm{~Hz}, 1 \mathrm{H}), 3.72(\mathrm{~s}, 6 \mathrm{H}), 3.15\left(\mathrm{dd}, J_{1}=4.0 \mathrm{~Hz}, J_{2}=12 \mathrm{~Hz}, 2 \mathrm{H}\right)$, $2.77(\mathrm{~m}, 1 \mathrm{H}), 2.34(\mathrm{~m}, 1 \mathrm{H})$.

${ }^{13} \mathrm{C}$ NMR $\left(100 \mathrm{MHz}, \mathrm{DMSO}-d_{6}\right): \delta=157.94,156.62,147.90$, $145.50,144.75,138.73,135.47,129.60,127.70,127.59,126.62$, $124.53,113.01,85.80,85.35,83.49,70.41,63.88,56.06,54.95$.

MS (ESI): $m / z[\mathrm{M}+\mathrm{H}]^{+}$calcd for $\mathrm{C}_{31} \mathrm{H}_{31} \mathrm{~N}_{4} \mathrm{O}_{6}: 555.2$; found: 555.3 .

5'-O-[Bis(4-methoxyphenyl)(phenyl)methyl]-2'-deoxy-xylo-inosine $(3)^{15}$

Product $2(0.554 \mathrm{~g}, 1.0 \mathrm{mmol})$ was added to a chilled stirred soln of Dess-Martin periodinane $(0.640 \mathrm{~g}, 1.5 \mathrm{mmol})$ in anhyd $\mathrm{CH}_{2} \mathrm{Cl}_{2}(8$ $\mathrm{mL}$ ) at $0{ }^{\circ} \mathrm{C}$, and the soln was stirred at $0{ }^{\circ} \mathrm{C}$ for $1 \mathrm{~h}$ and then at r.t. for 4 h. $i-\mathrm{PrOH}(8 \mathrm{~mL})$ was added and the resulting white slurry was cooled to $-45^{\circ} \mathrm{C}$. After $20 \mathrm{~min}$, freshly powdered $\mathrm{NaBH}_{4}(76 \mathrm{mg}$, $2.0 \mathrm{mmol}$ ) was added and the mixture was stirred for $12 \mathrm{~h}$ at $-45^{\circ} \mathrm{C}$. Acetone $(8 \mathrm{~mL})$ was added and the mixture was allowed to warm to r.t., diluted with $\mathrm{CH}_{2} \mathrm{Cl}_{2}(20 \mathrm{~mL})$, and washed sequentially with aq $\mathrm{NaHCO}_{3}(2 \times 20 \mathrm{~mL}), \mathrm{H}_{2} \mathrm{O}(2 \times 20 \mathrm{~mL})$, and brine $(2 \times 20 \mathrm{~mL})$. The organic layer was separated, dried $\left(\mathrm{Na}_{2} \mathrm{SO}_{4}\right)$, filtered, and concentrated under vacuum. The residue was purified by chromatography [silica gel, $\mathrm{CH}_{2} \mathrm{Cl}_{2}-\mathrm{EtOH}(15: 1)$ ] to give a white foamy solid; yield: $0.472 \mathrm{~g}(85.2 \%)$.

${ }^{1} \mathrm{H}$ NMR $\left(400 \mathrm{MHz}\right.$, DMSO- $d_{6}: \delta=12.39(\mathrm{~s}, 1 \mathrm{H}), 8.14(\mathrm{~s}, 1 \mathrm{H})$, $8.09(\mathrm{~d}, J=4 \mathrm{~Hz}, 1 \mathrm{H}), 7.41-6.77(\mathrm{~m}, 13 \mathrm{H}), 6.33\left(\mathrm{dd}, J_{1}=4.0 \mathrm{~Hz}\right.$, $\left.J_{2}=12 \mathrm{~Hz}, 1 \mathrm{H}\right), 5.42(\mathrm{~d}, J=4 \mathrm{~Hz}, 1 \mathrm{H}), 4.33(\mathrm{~m}, 1 \mathrm{H}), 4.21(\mathrm{dd}$, $\left.J_{1}=4 \mathrm{~Hz}, J_{2}=12 \mathrm{~Hz}, 1 \mathrm{H}\right), 3.73(\mathrm{~s}, 3 \mathrm{H}), 3.72(\mathrm{~s}, 3 \mathrm{H}), 3.18(\mathrm{dd}$, $\left.J_{1}=4.0 \mathrm{~Hz}, J_{2}=12 \mathrm{~Hz}, 2 \mathrm{H}\right), 2.72(\mathrm{~m}, 1 \mathrm{H}), 2.26(\mathrm{~m}, 1 \mathrm{H})$.

${ }^{13} \mathrm{C}$ NMR $\left(100 \mathrm{MHz}, \mathrm{DMSO}-d_{6}\right): \delta=157.96,156.60,147.73$, 145.76, 144.98, 138.78, 135.68, 135.54, 129.71, 127.68, 126.56, $124.02,113.01,85.42,83.93,83.01,69.28,63.14,54.94,40.82$.

MS (ESI): $m / z[\mathrm{M}+\mathrm{H}]^{+}$calcd for $\mathrm{C}_{31} \mathrm{H}_{31} \mathrm{~N}_{4} \mathrm{O}_{6}: 555.2$; found: 555.3 .

5'-O-[Bis(4-methoxyphenyl)(phenyl)methyl]-3'-O-mesyl-2'-deoxyinosine (4) ${ }^{16}$

A soln of $\mathrm{MsCl}(77 \mu \mathrm{L}, 1.0 \mathrm{mmol})$ in pyridine $(2 \mathrm{~mL})$ was added dropwise to a stirred soln of compound $3(0.277 \mathrm{~g}, 0.5 \mathrm{mmol})$ in anhyd pyridine $(4 \mathrm{~mL})$ at $0{ }^{\circ} \mathrm{C}$. The cooling bath was removed and the mixture was stirred for $12 \mathrm{~h}$ at r.t. The mixture was then poured into ice-water and stirred for another $10 \mathrm{~min}$. The resulting mixture was diluted with $\mathrm{CH}_{2} \mathrm{Cl}_{2}(20 \mathrm{~mL})$ and the organic layer was separated, washed with aq $\mathrm{NaHCO}_{3}(2 \times 20 \mathrm{~mL}), \mathrm{H}_{2} \mathrm{O}(2 \times 20 \mathrm{~mL})$, and brine $(2 \times 20 \mathrm{~mL})$ then dried $\left(\mathrm{Na}_{2} \mathrm{SO}_{4}\right)$, filtered, and concentrated under vacuum. The crude product was purified by chromatography [silica gel, $\left.\mathrm{CH}_{2} \mathrm{Cl}_{2}-\mathrm{EtOH}(20: 1)\right]$ to give a white foamy solid; yield: 0.139 $\mathrm{g}(44.0 \%)$

${ }^{1} \mathrm{H} \mathrm{NMR}\left(400 \mathrm{MHz}, \mathrm{CDCl}_{3}\right): \delta=11.91(\mathrm{~s}, 1 \mathrm{H}), 8.02(\mathrm{~s}, 1 \mathrm{H}), 7.99$ (s, $1 \mathrm{H}), 7.44-6.82(\mathrm{~m}, 13 \mathrm{H}), 6.43(\mathrm{~m}, 1 \mathrm{H}), 5.46(\mathrm{~m}, 1 \mathrm{H}), 4.37(\mathrm{~m}$, $1 \mathrm{H}), 3.80(\mathrm{~s}, 6 \mathrm{H}), 3.66\left(\mathrm{dd}, J_{1}=4.0 \mathrm{~Hz}, J_{2}=8 \mathrm{~Hz}, 1 \mathrm{H}\right), 3.36(\mathrm{dd}$, $\left.J_{1}=4.0 \mathrm{~Hz}, J_{2}=8 \mathrm{~Hz}, 1 \mathrm{H}\right), 2.93(\mathrm{~m}, 2 \mathrm{H}), 2.74(\mathrm{~s}, 3 \mathrm{H})$.

${ }^{13} \mathrm{C}$ NMR $\left(100 \mathrm{MHz}, \mathrm{CDCl}_{3}\right): \delta=153.44,153.24,142.34,140.10$ $139.41,135.22,130.63,130.52,124.79,122.86,122.62,121.61$, $119.99,107.91,81.36,79.11,78.85,65.71,57.10,49.97,35.83$, 24.47.

MS (ESI): $m / z[\mathrm{M}+\mathrm{H}]^{+}$calcd for $\mathrm{C}_{32} \mathrm{H}_{33} \mathrm{~N}_{4} \mathrm{O}_{8} \mathrm{~S}: 633.2$; found: 633.3.

\section{5'-O-[Bis(4-methoxyphenyl)(phenyl)methyl]-2-deoxy-3'-thio-} inosyl $3^{\prime}-S$-Acetate (5) ${ }^{16}$

$\mathrm{KSAc}(70.3 \mathrm{mg}, 0.617 \mathrm{mmol})$ was added to a colorless soln of compound $4(0.130 \mathrm{~g}, 0.21 \mathrm{mmol})$ in anhyd DMF $(8 \mathrm{~mL})$, and the mix- ture was stirred at $60^{\circ} \mathrm{C}$ for $24 \mathrm{~h}$ under argon, The mixture was then transferred into $\mathrm{CH}_{2} \mathrm{Cl}_{2}(10 \mathrm{~mL})$ and the soln with washed with aq $\mathrm{NaHCO}_{3}(2 \times 20 \mathrm{~mL}), \mathrm{H}_{2} \mathrm{O}(2 \times 20 \mathrm{~mL})$, and brine $(2 \times 20 \mathrm{~mL})$. The organic layer was separated, dried $\left(\mathrm{Na}_{2} \mathrm{SO}_{4}\right)$, filtered, concentrated, and co-evaporated with toluene to remove the DMF. The residual oil was purified by chromatography [silica gel, $\mathrm{CH}_{2} \mathrm{Cl}_{2}-\mathrm{EtOH}$ $(15: 1)]$ to give a white foamy solid; yield: $86.6 \mathrm{mg}(67.4 \%)$.

${ }^{1} \mathrm{H}$ NMR $\left(400 \mathrm{MHz}, \mathrm{CDCl}_{3}\right.$ ): $\delta=12.13$ (s, $\left.1 \mathrm{H}\right), 8.07$ (s, $\left.1 \mathrm{H}\right), 7.99$ $(\mathrm{s}, 1 \mathrm{H}), 7.44-6.77(\mathrm{~m}, 13 \mathrm{H}), 6.33\left(\mathrm{dd}, J_{1}=4.0 \mathrm{~Hz}, J_{2}=8 \mathrm{~Hz}, 1 \mathrm{H}\right)$, $4.27(\mathrm{~m}, 1 \mathrm{H}), 4.17\left(\mathrm{dd}, J_{1}=4 \mathrm{~Hz}, J_{2}=8 \mathrm{~Hz}, 1 \mathrm{H}\right), 3.78(\mathrm{~s}, 6 \mathrm{H})$, $3.40(\mathrm{~d}, J=4.0 \mathrm{~Hz}, 2 \mathrm{H}), 3.03(\mathrm{~m}, 1 \mathrm{H}), 2.56(\mathrm{~m}, 1 \mathrm{H}), 2.34(\mathrm{~s}, 3 \mathrm{H})$.

${ }^{13} \mathrm{C}$ NMR $\left(100 \mathrm{MHz}, \mathrm{CDCl}_{3}\right): \delta=194.04,159.21,158.53,148.42$, $144.99,144.39,139.48,138.39,135.57,130.09,129.15,128.15$, $127.84,126.93,125.14,113.17,86.64,84.48,84.02,63.29,55.25$, $40.80,39.61,30.62$.

MS (ESI): $m / z[\mathrm{M}+\mathrm{H}]^{+}$calcd for $\mathrm{C}_{33} \mathrm{H}_{33} \mathrm{~N}_{4} \mathrm{O}_{6} \mathrm{~S}: 613.2$; found: 613.3.

\section{5'-O-[Bis(4-methoxyphenyl)(phenyl)methyl]-2-deoxy-3'-thio-} inosine (6)

A suspension of $\mathrm{LiAH}_{4}(15.2 \mathrm{mg}, 0.4 \mathrm{mmol})$ in anhyd THF $(5 \mathrm{~mL})$ was cooled to $0{ }^{\circ} \mathrm{C}$ and then a soln of compound $5(61.2 \mathrm{mg}, 0.1$ $\mathrm{mmol})$ in THF $(5 \mathrm{~mL})$ was added dropwise under argon. The mixture was stirred for $4 \mathrm{~h}$ at r.t. then treated with $1 \mathrm{M}$ aq HOAc $(2 \times$ $20 \mathrm{~mL}) . \mathrm{CH}_{2} \mathrm{Cl}_{2}(10 \mathrm{~mL})$ was added and the organic layer was separated, washed with brine $(2 \times 20 \mathrm{~mL})$, dried $\left(\mathrm{Na}_{2} \mathrm{SO}_{4}\right)$, filtered, and concentrated under vacuum. The residue was purified by chromatography [silica gel, $\mathrm{CH}_{2} \mathrm{Cl}_{2}-\mathrm{EtOH}$ (12:1)] to give a white foamy solid; yield: $52.2 \mathrm{mg}(91.5 \%)$.

${ }^{1} \mathrm{H}$ NMR $\left(400 \mathrm{MHz}, \mathrm{CDCl}_{3}\right): \delta=12.62(\mathrm{~s}, 1 \mathrm{H}), 8.10(\mathrm{~s}, 1 \mathrm{H}), 8.04$ $(\mathrm{s}, 1 \mathrm{H}), 7.43-6.80(\mathrm{~m}, 13 \mathrm{H}), 6.32\left(\mathrm{dd}, J_{1}=4.0 \mathrm{~Hz}, J_{2}=8 \mathrm{~Hz}, 1 \mathrm{H}\right)$, $4.00(\mathrm{~m}, 1 \mathrm{H}), 3.78(\mathrm{~s}, 6 \mathrm{H}), 3.75-3.70(\mathrm{~m}, 1 \mathrm{H}), 3.53\left(\mathrm{dd}, J_{1}=\right.$ $\left.4.0 \mathrm{~Hz}, J_{2}=8 \mathrm{~Hz}, 1 \mathrm{H}\right), 3.42\left(\mathrm{dd}, J_{1}=4.0 \mathrm{~Hz}, J_{2}=8 \mathrm{~Hz}, 1 \mathrm{H}\right), 2.93$ $(\mathrm{m}, 1 \mathrm{H}), 2.49(\mathrm{~m}, 1 \mathrm{H}), 1.65(\mathrm{~d}, J=8 \mathrm{~Hz}, 1 \mathrm{H})$.

${ }^{13} \mathrm{C} \mathrm{NMR}\left(100 \mathrm{MHz}, \mathrm{CDCl}_{3}\right): \delta=158.21,157.53,147.16,143.99$, $143.38,137.59,134.52,129.01,127.07,126.93,125.94,124.21$, $112.21,87.39,85.61,83.15,60.75,54.22,41.68,34.01$.

MS (ESI): $m / z[\mathrm{M}+\mathrm{H}]^{+}$calcd for $\mathrm{C}_{31} \mathrm{H}_{30} \mathrm{~N}_{4} \mathrm{O}_{5} \mathrm{~S}$ : 571.2; found: 571.3 .

$S$-[5'-O-[Bis(4-methoxyphenyl)(phenyl)methyl]-2-deoxy-3'thioinosinyl] $O$-(2-Cyanoethyl) Diisopropylamidothiophosphite (7)

DIPEA $(68.4 \mu \mathrm{L}, 0.40 \mathrm{mmol})$ and $i-\mathrm{Pr}_{2} \mathrm{NP}(\mathrm{Cl})\left(\mathrm{CH}_{2}\right)_{2} \mathrm{CN}(31.8 \mu \mathrm{L}$, $0.14 \mathrm{mmol}$ ) were added to a stirred soln of compound $6(40 \mathrm{mg}$, $0.070 \mathrm{mmol})$ in anhyd $\mathrm{CH}_{2} \mathrm{Cl}_{2}(5 \mathrm{~mL})$, and the mixture was stirred at r.t. for $3 \mathrm{~h}$. The mixture was then diluted with $\mathrm{CH}_{2} \mathrm{Cl}_{2}(10 \mathrm{~mL})$ and washed successively with $\mathrm{H}_{2} \mathrm{O}(2 \times 20 \mathrm{~mL})$ and brine $(2 \times 20$ $\mathrm{mL})$ then dried $\left(\mathrm{Na}_{2} \mathrm{SO}_{4}\right)$, filtered, and concentrated under vacuum. The residue was purified by chromatography (silica gel, $0.5 \% \mathrm{Et}_{3} \mathrm{~N}$ in $\left.5 \% \mathrm{EtOH}-\mathrm{CH}_{2} \mathrm{Cl}_{2}\right)$ to give a white solid; yield: $51.3 \mathrm{mg}(95.2 \%$ yield).

${ }^{1} \mathrm{H}$ NMR $\left(400 \mathrm{MHz}, \mathrm{CDCl}_{3}\right): \delta=13.17(\mathrm{~s}, 1 \mathrm{H}), 8.15-8.06(\mathrm{~s}, 2 \mathrm{H})$, 7.40-6.76 (m, $13 \mathrm{H}), 6.39-6.29(\mathrm{~m}, 1 \mathrm{H}), 4.54-4.44(\mathrm{~m}, 1 \mathrm{H})$, 4.24-4.17 (m, $1 \mathrm{H}), 3.76(\mathrm{~s}, 6 \mathrm{H}), 3.66-3.52(\mathrm{~m}, 4 \mathrm{H}), 3.48-3.36(\mathrm{~m}$, $2 \mathrm{H}), 3.04-2.95(\mathrm{~m}, 1 \mathrm{H}), 2.75-2.66(\mathrm{~m}, 1 \mathrm{H}), 2.60-2.55(\mathrm{~m}, 1 \mathrm{H})$, $2.45-2.41(\mathrm{~m}, 1 \mathrm{H}), 1.28-1.02(\mathrm{~m}, 12 \mathrm{H})$.

${ }^{13} \mathrm{C}$ NMR $\left(100 \mathrm{MHz}, \mathrm{CDCl}_{3}\right): \delta=158.74,158.54,148.60,145.28$, $144.47,138.37,135.61,130.06,128.14,127.87,126.94,125.13$, $117.48,113.15,86.49,84.57,73.47,63.33,55.35,55.25,45.92$, $43.27,29.69,24.62,20.19$.

${ }^{31} \mathrm{P}$ NMR $\left(162 \mathrm{MHz}, \mathrm{CDCl}_{3}\right)$ : $\delta=165.42,165.05$.

HRMS: $m / z[\mathrm{M}]^{+}$calculated for $\mathrm{C}_{40} \mathrm{H}_{47} \mathrm{~N}_{6} \mathrm{O}_{6} \mathrm{PS}$ : 770.3015; found: 770.3038 . 


\section{Acknowledgment}

We thank the National Nature Science Foundation of China (No. 20702014) and the Fundamental Research Funds for the Central Universities of China (WK1114053) for their financial support. We are grateful to Professor H. Tian of the East China University of Science and Technology for his valuable support for this project.

Supporting Information for this article is available online at http://www.thieme-connect.com/ejournals/toc/synthesis.

\section{References}

(1) Piccirilli, J. A.; Vyle, J. S.; Caruthers, M. H.; Cech, T. R. Nature (London) 1993, 361, 85.

(2) (a) Thibaudeau, C.; Plavec, J.; Garg, N.; Papchikhin, A.; Chattopadhyaya, J. J. Am. Chem. Soc. 1994, 116, 4038. (b) Plavec, J.; Thibaudeau, C.; Chattopadhyaya, J. J. Am. Chem. Soc. 1994, 116, 6558

(3) (a) Liu, X.; Reese, C. B. Tetrahedron Lett. 1996, 37, 925. (b) Weinstein, L. B.; Earnshaw, D. J.; Cosstick, R.; Cech, T. R. J. Am. Chem. Soc. 1996, 118, 10341.

(4) Gordon, P. M.; Sontheimer, E. J.; Piccirilli, J. A. RNA 2000, 6, 199.

(5) Szewczak, A. A.; Kosek, A. B.; Piccirilli, J. A.; Strobel, S. A. Biochemistry 2002, 41, 2516.

(6) (a) Butora, G.; Kenski, D. M.; Cooper, A. J.; Fu, W.; Qi, N.; Li, J. J.; Flanagan, W. M.; Davies, I. W. J. Am. Chem. Soc. 2011, 133, 16766. (b) Kannan, A.; Burrows, C. J. J. Org. Chem. 2010, 76, 720. (c) Wunderlich, C. H.; Spitzer, R.; Santner, T.; Fauster, K.; Tollinger, M.; Kreutz, C. J. Am. Chem. Soc. 2012, 134, 7558 .
(7) Beevers, A. P. G.; Fettes, K. J.; O’Neil, I. A.; Roberts, S. M.; Arnold, J. R. P.; Cosstick, R.; Fisher, J. Chem. Commun. (Cambridge) 2002, 1458.

(8) (a) Sabbagh, G.; Fettes, K. J.; Gosain, R.; O’Neil, I. A.; Cosstick, R. Nucleic Acids Res. 2004, 32, 495. (b) Ariza, X.; Bou, V.; Vilarrasa, J. J. Am. Chem. Soc. 1995, 117, 3665. (c) Terrazas, M.; Ariza, X.; Farràs, J.; Guisado-Yang, J. M.; Vilarrasa, J. J. Org. Chem. 2004, 69, 5473. (d) Terrazas, M.; Ariza, X.; Vilarrasa, J. Org. Lett. 2005, 7, 2477.

(9) Lu, J.; Li, N.-S.; Sengupta, R. N.; Piccirilli, J. A. Bioorg. Med. Chem. 2008, 16, 5754.

(10) (a) Martin, F. H.; Castro, M. M.; Aboul-ela, F.; Tinoco, I. Nucleic Acids Res. 1985, 13, 8927. (b) Kawase, Y.; Iwai, S.; Inoue, H.; Miura, K.; Ohtsuka, E. Nucleic Acids Res. 1986, 14, 7727. (c) Ohtsuka, E.; Matsuki, S.; Ikehara, M.; Takahashi, Y.; Matsubara, K. J. Biol. Chem. 1985, 260, 2605.

(11) Case-Green, S. C.; Southern, E. M. Nucleic Acids Res. 1994, 22, 131.

(12) Challa, H.; Bruice, T. C. Bioorg. Med. Chem. 2004, 12, 1475

(13) Hansske, F.; Madej, D.; Robins, M. J. Tetrahedron 1984, 40, 125.

(14) (a) Bae, S.; Lakshman, M. K. J. Am. Chem. Soc. 2007, 129, 782. (b) Seela, F.; Kaiser, K. Nucleic Acids Res. 1986, 14, 1825. (c) Hansen, A. S.; Thalhammer, A.; El-Sagheer, A. H.; Brown, T.; Schofield, C. J. Bioorg. Med. Chem. Lett. 2011, 21, 1181. (d) Lakshman, M. K.; Bae, S. WO 2008045535 2008; Chem. Abstr. 2008, 148, 449867.

(15) Eisenhuth, R.; Richert, C. J. Org. Chem. 2008, 74, 26.

(16) Xiao, P.; Bai, Y.; Chen, J.; Lu, Z. CN 102180926 2011; Chem. Abstr. 2011, 155, 484417. 